Supplement of Geosci. Model Dev., 9, 3093-3110, 2016

http://www.geosci-model-dev.net/9/3093/2016/

doi:10.5194/gmd-9-3093-2016-supplement

(c) Author(s) 2016. CC Attribution 3.0 License.

(c) (i)

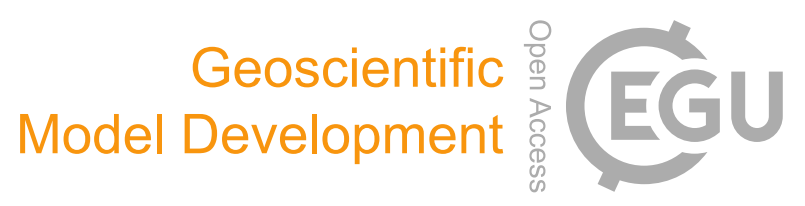

Supplement of

\title{
Community Intercomparison Suite (CIS) v1.4.0: a tool for intercomparing models and observations
}

Duncan Watson-Parris et al.

Correspondence to: Duncan Watson-Parris (duncan.watson-parris@physics.ox.ac.uk)

The copyright of individual parts of the supplement might differ from the CC-BY 3.0 licence. 


\section{CIS Reference Card}

Community Intercomparison Suite v1.4.0

August 2016 - www.cistools.net

Global options

Some options apply to all commands:

-o Specify an output filename.

? $\quad$ Print help about the command

-q Quiet - suppress all output to screen

-v $\quad$ Verbose - include more detailed screen output

Commands

The basic structure of a CIS command is:

\$ cis <command $><$ variable $>$ :<file $>$ :<options $><$ options $>$

The $<$ data variable $>$ : $<$ data file $>$ construct is common to all commands and is generally referred to as a 'datagroup'. A datagroup can contain multiple, comma separated, variables, and filenames, and both can include wildcards. All datagroups accept the product='...' option to specify the plugin to read the data with.

All currently available commands are summarised below.

version

Will print the version of CIS currently being used.

subset <data variable $>:<$ data file $><$ subset limits $>$

Subset any number of datasets to the specified spatio-temporal extent specified by a comma separated sequence of one or more coordinate ranges of the form

variable $=[$ start,end]. The variable can be its name as it is in the data file, its CF standard name, or an axes name shorthand such as $x, y, t, z$ and $p$.

aggregate <data variable>:<data file>:<options> <aggregation grid>

Aggregate a dataset over one or more coordinates, either completely, or to a user defined grid. Multiple coordinates should be comma separated, grids are defined using coordinate $=[$ start,end, step $]$.

\section{col $<$ data variable $>$ :<data file $><$ sample file $>$ :<colocation method $>$}

Collocate the data variable onto the spatio-temporal sampling provided by the sample file. Available collocation methods depend on the data-types:

\begin{tabular}{|c|c|c|}
\hline & GRIDDED & UNGRIDDED \\
\hline GRIDDED & lin, nn, box & lin, $n n$ \\
\hline UNGRIDDED & box, bin & box \\
\hline
\end{tabular}

Where the default options are shown in bold and described below.

lin linear interpolation in space and time

nn Nearest Neighbour

bin operates kernel on all source data in sample bounds

box operates kernel on all source data in user-defined box on sample

\section{eval <data variable $>$ :<data file $><$ expression $><$ units $>$}

Perform an evaluation over correlated data variables of any valid Python expression. Aliases can be used to simplify evaluation expressions as shown in the recipes.

stats < data variable $>$ :<data file $>$

Calculate statistics and correlation factors between exactly two correlated datasets.

info <data file $>[-v<$ data variable $>]$

Print the variables present in a file, or set of files, and optionally summary information fo one or more specific variables.

plot $<$ data variable $>:<$ data file $>$ :<layer options $>$ [plot options]

Plot any CIS compatible data using a wide variety of plot types and options. Each layer corresponds to a single variable, so only one variable is allowed per datagroup.

Layer options

Multiple layer options should be specified in a comma separated list.

color Colour of markers, e.g. for scatter plot points or contour lines

cmap Colour map to use, e.g. for contour lines or heatmap

cmin The minimum value for the colourmap

\begin{tabular}{l|l} 
cmax & The maximum value for the colourmap
\end{tabular}

edgecolor Colour of scatter marker edges

itemstyle Shape of scatter marker

label Name of datagroup for the legend

contnlevels Additional datagroup options for contour plots only:

contlevels The number of levels for the contour plot 
contlabel

A list of levels for the contour plot, e.g. contlevels $=[0,1,3,10]$

contwidth

Options are true or false, if true then contour labels are shown

contfontsize

type

Width of the contour lines

The type of plot for this particular layer (only used for overlay plots)

Plot options

\begin{tabular}{|c|c|}
\hline --type & $\begin{array}{l}\text { The plot type, one of: line, scatter, heatmap, contour, contourf, } \\
\text { histogram } 2 d \text {, histogram } 3 d \text {, comparativescatter and overlay }\end{array}$ \\
\hline --xlabel & The label for the $x$ axis \\
\hline --ylabel & The label for the y axis \\
\hline --cbarlabel & The label for the colour bar \\
\hline --xtickangle & The angle for the ticks on the $x$ axis \\
\hline --ytickangle & The angle for the ticks on the $y$ axis \\
\hline --title & The title of the plot \\
\hline --itemwidth & $\begin{array}{l}\text { The width of an item. Unit are points in the case of a line, and } \\
\text { points squared in the case of a scatter point }\end{array}$ \\
\hline --fontsize & The size of the font in points \\
\hline --cmap & The colour map to be used when plotting a 3D plot \\
\hline --height & The height of the plot, in inches \\
\hline --width & The width of the plot, in inches \\
\hline --xbinwidth & The width of the histogram bins on the $x$ axis \\
\hline --ybinwidth & The width of the histogram bins on the $y$ axis \\
\hline --cbarorient & The orientation of the colour bar, either horizontal or vertical \\
\hline --nocolourbar & Hides the colour bar on a 3D plot \\
\hline --grid & Shows grid lines \\
\hline --plotwidth & Width of the plot in inches \\
\hline --plotheight & Height of the plot in inches \\
\hline --cbarscale & $\begin{array}{l}\text { Used to change the size of the colour bar when plotting, defaults } \\
\text { to } 0.55 \text { for vertical colour bars, } 1.0 \text { for horizontal. }\end{array}$ \\
\hline --coastlinescolour & The colour of the coastlines on a map \\
\hline --nasabluemarble & Use NASA Blue Marble for the background \\
\hline$--\log x$ & The $x$ axis will be plotted using a log scale of base 10 \\
\hline$--\log y$ & The $y$ axis will be plotted using a log scale of base 10 \\
\hline$--\log v$ & The values (colours) will be plotted using a log scale of base 10 \\
\hline
\end{tabular}

Also, the arguments --xmin, --xmax, --xstep, --ymin, --ymax, --ystep, --vmin, --vmax and

--vstep can be used to specify the range of values to plot on each axis.

Recipes

There are some useful command structures which may not be immediately obvious, some of those are listed here.
Reading model fields with ancillary orography files

Many model outputs include an orography in a separate ancillary file, assuming CF-

compliant standard names, these can easily be included to produce correct altitude fields

$\$$ cis plot aot500aer:aot500aer.nc,orography.nc

Plotting multiple variables

Different plot types handle plotting multiple variables slightly differently, which may lead to some confusion.

For plotting multiple variables in line and scatter plots, simply use as many datagroups as is necessary, applying layer options as needed:

$\$$ cis plot CCN1:file.nc:label='CCN at 1\%' CCN2:file.nc:label='CCN at 2\%'

For plotting multiple variables over each other, on their own scales, use an overlay plot: \$ cis plot CCN1:file.nc:type=heatmap CCN2:file.nc:type=contour
--type=overlay

Calculating the difference between two datasets

A useful demonstration of the use of eval is in calculating the difference between two collocated files:

\$ cis eval var1=a:obs.nc var2=b:collocated_model.nc "a-b" 1

-o var_diff:obs_minus_model.nc

Notice here we have used aliases for the variable names, and also specified the output variable name (this only works for the eval command currently)

Masking values based on a second variable

A more advanced example of eval is masking values from one variable based on the value of a second variable. For example only including the mean values if the number of points used for that mean was more than 20 :

\$ cis eval ccn,ccn_num_points=num:collocated_ccn.nc

"numpy.ma.masked_where(num<20, ccn)" 1 -o nc:masked.nc

CIS as a Python Library

Using $\mathrm{ClS}$ to read datasets into Python, and even convert to Pandas, is straightforward:

from cis import read_data

data = read_data ('my_file.nc', 'my_var')

$\mathrm{df}=$ data.as_data_frame() 